\title{
Novel Tyrosine Kinase Inhibitors in the Treatment of Cancer
}

\section{Alberto Ocana, Rosario Serrano, Raul Calero and Atanasio Pandiella}

Tyrosine kinases (TKs) are involved in different cellular functions including extracellular signalling, intracellular communication, proliferation, cell cycle control or apoptosis/survival regulation. As most of them control physiologic functions in nontransformed cells their deregulation produces important cellular changes linked to an oncogenic phenotype. A clear example is the oncogenic role of the epidermal growth factor receptor (EGFR) family in different tumor types. However, many other tyrosine kinases have been linked to cancer including citoplasmic kinases like SRC and FAK, and membrane receptors such as the Vascular Endothelial Growth Factor receptor (VEGFR), the Insulin-like Growth Factor-I receptor (IGF-IR) or the Hepatocyte Growth Factor receptor (MET), among others. Dysfunction of receptor tyrosine kinases (RTKs) can be produced by different mechanisms including abnormal activation by ligand binding, overexpression or structural alterations like mutations or truncations. Examples of this abnormal activation can be observed in breast cancer with HER2 overexpression or in lung cancer with EGFR mutations. The continued kinase activation produces a gain of function that results in proliferation, survival and in some circumstances resistance to anticancer chemotherapies. As a consequence of their implication in tumorigenic processes, different strategies were developed to inhibit the pathologic activation of these proteins. For RTKs several strategies were considered, but only two of them have successfully reached the clinic: (i) monoclonal antibodies against the extracellular domain or (ii) tyrosine kinase inhibitors. Clear examples for the first group are the antibody trastuzumab against the HER2 receptor or the antibody cetuximab against the EGF receptor. Antibody binding produces a down-regulation of the receptor, decreasing the oncogenic stimuli. In the cases were activation of the RTK was produced by mutations or by a constitutive activated intracellular form; extracellular antibodies were not a reasonable strategy. Similarly, cytoplasmic kinases were not druggable with monoclonal antibodies.

Small tyrosine kinase inhibitors were designed to affect ATP-binding in the kinase domain producing their effect in a competitive manner with the ATP. Recently, some other allosteric inhibitors, not directly acting on the ATP binding loop have been described. Imatinib mesylate was the first TK inhibitor developed against the fusion activated oncogenic protein kinase bcr-abl in Chronic Myeloid Leukemia. Later, two small RTK inhibitors, erlotinib and gefitinib were developed against the EGF receptor. Treatment with these drugs demonstrated efficacy in several tumor types including lung cancer patients with EGFR mutations. The activity of these drugs is limited and most of the patients do not respond or progress in a short period of time. The first mechanism described for primary resistance in lung cancer and chronic myeloid leukemia was related to secondary mutations. Other mechanism described latter was the activation of alternative RTK pathways. For instance, in HER2 overexppressing breast cancer cells, tyrosine kinase inhibitors prevent the autophosphorylation of EGFR and HER2 but inefficiency and transitory inhibit the phosphorylation of HER3. In lung cancer, similar findings have shown that resistant tumors to tyrosine kinase inhibitors have a constitutive activation of HER3 secondary to an amplification of the MET receptor. In this context, the presence of other activated RTKs in the tumor could represent an important

mechanism of resistance to TK inhibitors. These observations led to hypothesize that co-targetting of RTKs could be a better therapeutic strategy. Indeed, concomitant inhibition of several RTKs increases the efficacy of single target inhibition.

Multi-tyrosine kinase inhibitors were developed to target multiple TK in an unselected manner. Compounds such as Sorafenib or Sunitinib inhibited several kinases involved in the control of different biological functions. For instance, Sorafenib and Sunitinib inhibit the VEGFR and Sorafenib also inhibits the B-Raf isoform. Both TK inhibitors showed antitumor activity in kidney cancer with an increase in overall outcome. Similarly Sorafenib produced a survival benefit for patients with hepatocarcinoma.

The toxicity profile of these compounds is related to the group of kinases inhibited by each agent. For TK inhibitors that target receptors involved in angiogenesis like VEGFR, hemorrhagic risk, thrombosis, or hypertension are toxicities that maybe considered. For those that target the EGFR the main toxicities are diarrhoea or skin rash. Interestingly, as toxicity is a sign of the target inhibition, several studies have evaluated these side effects as a surrogate marker for the drug activity.

Two groups of TK inhibitors are currently under clinical development: (i) specific multi-TK inhibitors and (ii) irreversible TK inhibitors. At present TK inhibitors are being designed to target specific TK proteins involved in oncogenic processes. This is the case of the anti-TK inhibitor ZD6474 that selectively inhibits the EGF and VEGF receptors, the TK inhibitor AEE788 that inhibit EGFR, HER2 and VEGFR; or the TK inhibitor AXL184 that inhibits MET, RET and VEGFR. Irreversible TK compounds including EKB-569, HKI-272, 
CL-387,785 and CI-1033 are currently being tested in solid tumors in which secondary mutations or even primary mutations limit the efficacy of reversible TK inhibitors. This is the case of lung

cancer patients with resistance to erlotinib and gefitinib. Furthermore, irreversible TK inhibitors have shown to be more effective in other tumor types in which no mutations are found and activation is produced by an ErbB overexpression as occurs with HER2 in breast cancer.

Along the way, we have also realized that downstream oncogenes could modify the response to TK inhibitors. This is the case for the inefficacy of anti EGFR therapies in colon cancer when K-RAS mutation is present. In a similar manner, Lapatinib an anti EGFR/HER2 TK inhibitor has shown limited activity in breast cancer models in which downstream PI3K mutations exist. Bearing this in mind, future TK inhibitors should be developed against these oncogenic alterations, or at least be given only when no downstream mutations are found. In summary, multi-TK inhibitors are being designed to target multiple activated kinases. The future design plan for these compounds should take into consideration the activated array of kinases in a given tumor. This undoubtedly will increase the efficacy, also avoiding toxicity. Furthermore, irreversible TK inhibitors show more efficacy than reversible ones, with a similar toxicity profile, thereby presenting these inhibitors as attractive alternatives to reversible inhibitors. In addition, irreversible inhibitors may offer advantages with respect to their ability to be more efficacious to fight avoid resistance due to secondary mutations. The development of multikinase, reversible, irreversible, and other allosteric inhibitors of tyrosine kinases is clearly widening the armamentarium to fight neoplastic diseases, and will allow better targeting of the tumoral cells, improved toxicological properties, and more favourable responses by preventing drug resistance. 